\title{
The Effects of Scalloping Width and Position on Jet Mixing of Lobed Nozzles
}

\author{
Liu DaWei¹, Huang Jun¹, Sheng ZhiQiang ${ }^{1}$, Ji Jinzu¹
}

\begin{abstract}
A series of geometric models of lobed nozzles with a central plug was created by different scalloping positions and widths. The shapes of lower and upper edges, cutting depth were kept unchanged. In this study, by the use of numerical simulation, the effects of scalloped width and position on the performance of jet mixing in the pumping condition were analyzed. The results indicated that, as the position of scalloped lower edge kept constant, the radial position of the accelerated mixing region of sidewalls did not change. The accelerated mixing region is enlarged as scalloped width increased, while the growth rate of enlarged region is less than the growth rate of scalloping width. When the scalloped region with the same width moved outward in the radial direction, the mixing rate of the region of the lobe outer edges increased. However, the distance for complete mixing of the primary stream in the core region was increased. It was also found that inward moving of scalloped lower edge enhanced the effect of strengthening streamwise vortices but induced more pressure loss.
\end{abstract}

KEYWORDS: Lobed nozzles, Scalloping, Thermal mixing efficiency, Streamwise vorticity, Numerical simulation.

\section{INTRODUCTION}

Lobed nozzles can increase the interface area of primary and secondary flow (Elliott et al. 1992) and the interaction between streamwise and normal vorticity can enhance the mixing between primary and secondary flow (Povinelli and Anderson 1984; McCormick and Bennett 1994). Such ejector schemes provide a simple mean of reducing jet noise by mixing high velocity primary flow with cool ambient air which can also provide a thrust gain (Presz et al. 1994; Acheson et al. 2011). For the military infrared stealth purpose, the lobed nozzles can dilute the engine plume with cold ambient air, decrease $\mathrm{CO}_{2}$ concentration and temperature; therefore it reduces the target detectability (Pan et al. 2013; Zhang et al. 2014). Meanwhile, from the fuel savings, full fuel combustion can be achieved by enhancing the mixing of reactants while delaying ignition in a controlled manner (Smith et al. 1997; Mitchell et al. 2004). Previous studies show that the scalloping of lobed nozzles can improve the mixing performance (Presz et al. 1994; Abolfadl and Sehra 1993; Yu et al. 1997, 2000; Hu et al. 1999; Mao et al. 2009; Lei 2011) but cause some extra total pressure loss (Hu et al. 1999). Presz et al. (1994) found that the scalloping could generate additional vortices, which are upstream the lobe exit plane. The scalloped lobes with large angle can generate benefits like lower wall friction loss and more rapid mixing. Then a short compact ejector is feasible. Yu attributed the enhancement to that, and the streamwise vorticity is stronger because of some additional streamwise vortices formed due to the scalloping (Yu et al. 1997). Through the scalloping of sword alternating lobed nozzle, Sheng et al. (2014) found that the degree of scalloping was increased, and the mixing in the downstream region of the lobe peak was accelerated; however, the primary flow in

\footnotetext{
三 1.Beijing University of Aeronautics and Astronautics - School of Aeronautic Science and Engineering - Beijing - China.

Author for correspondence: Liu Dawei | Beijing University of Aeronautics and Astronautics - School of Aeronautic Science and Engineering | Beijing 10019 - China Email: buaapebble@sina.com

Received: 07/27/2015 | Accepted: 09/30/2015
} 
center needed larger mixing length. Lei found that the overall streamwise circulation was not necessarily increased after scalloping. In an experimental investigation of a scalloped mixer, Lei (2011) showed that the overall streamwise circulation was not necessarily increased after scalloping. The more rapidly decaying streamwise vorticity presented a more effective mixing process because of smaller-scale but more developed vortices (Lei 2011; Lei et al. 2012; Wright et al. 2013).

All the previous works on scalloped lobed nozzles were concentrated on large lobe penetration angles lobe nozzles. However, in practical applications of helicopter or warship, designers have preferred small angle lobed nozzles. Because the diameter of mix tube is limited, small lobe penetration angles can penetrate the sidewalls of mix tube. Large angle lobe may cause the primary stream impacting the sidewalls, then the temperature of sidewalls and the pressure loss increase. Also, no parametric studies were performed on these previous studies; in general, scalloping parameters like width, depth, shape, and position were coupled together. In this work, the effects of scalloping width and position on the jet mixing in pumping condition were analyzed numerically. To achieve this, a series of small angle lobed nozzle models with a central plug and different scalloping positions and widths were used. The lower and upper edges and cutting depth were kept unchanged.

\section{INVESTIGATED CONFIGURATIONS}

The geometry model of lobed nozzles with a central plug (PLN) and mixing tube are illustrated in Fig. 1. The abbreviations for diameter and radius are $D$ and $R$, respectively. The annular entrance of the nozzle is formed by two circles with diameters of 210 and $400 \mathrm{~mm}$. The inward and outward lobe penetration angles are $12.9^{\circ}$ and $12.1^{\circ}$, respectively. The length of mixing duct is $1,150 \mathrm{~mm}$ and its entrance is $100 \mathrm{~mm}$ ahead of the exit of the nozzle. The diameter D of the duct is $700 \mathrm{~mm}$, and the length-to-diameter ratio of the mixing segment $\mathrm{L} / \mathrm{D}$ is 1.5 .

Figure 2 shows the scalloped lobed nozzles which are investigated in this paper, and the geometrical dimensions for each scalloping are shown in Fig. 3. The scalloped lobed nozzles $\mathrm{N}^{\circ} 1,2$, and 3, i.e. scalloping $\mathrm{N}^{\circ} 1,2$, and 3 (S1, S2, and S3), have the same cut shape, but with the radial cut position moving towards outside in an equal distance. Scallopings $\mathrm{N}^{\circ} 4$ and 5

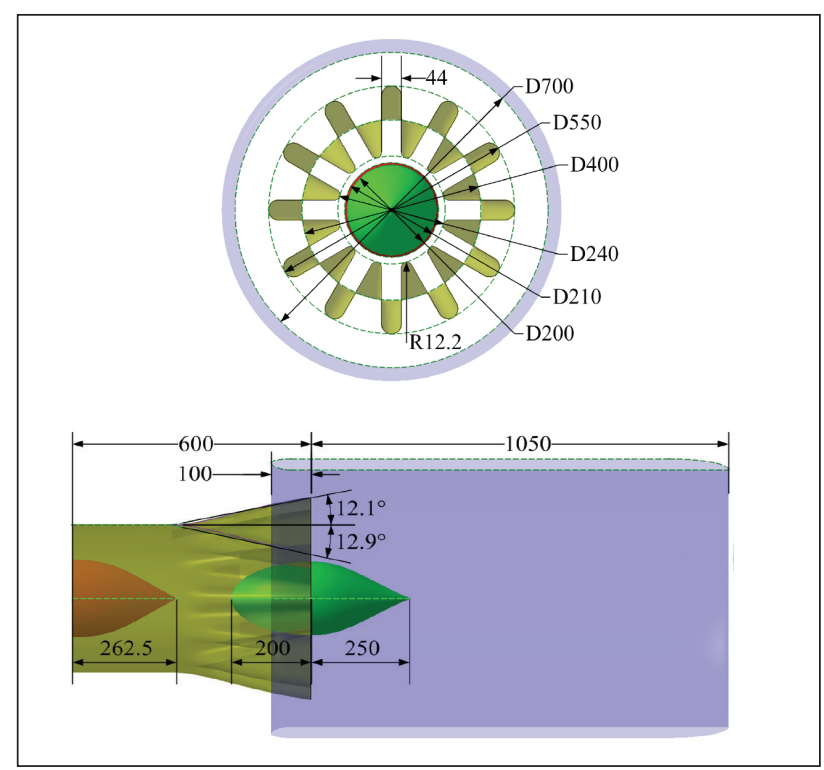

Figure 1. Geometry dimensions of the baseline lobed mixer (all dimensions are in $\mathrm{mm}$ ).

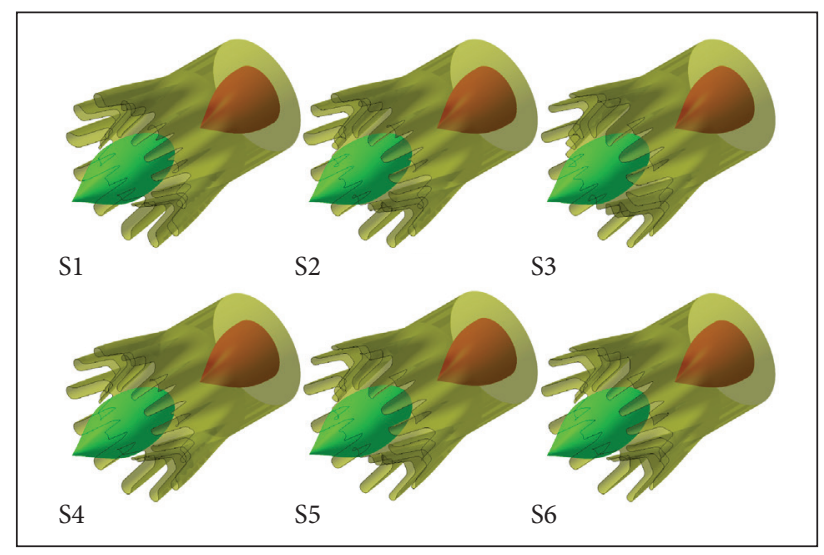

Figure 2. The scalloped lobed nozzles schemes.

(S4 and S5) also have the same cut shape, and the lower and upper edges of S4 lap over with the lower edge of S1 and the upper edge of S2, and those of S5 lap over with the lower edge of S2 and the upper edge of S3. Scalloping No. 6 (S6) has the largest cut width with the lower and upper edges lapping over with the lower edge of $S 1$ and the upper edge of $S 3$, respectively.

\section{CFD MODEL}

The numerical simulation domain and grids are illustrated in Fig. 4. Owing to the complicated geometry shapes, tetrahedral cells were adopted to discretize the whole flow-field domain. Three boundary layer cells were used and the first layer height 


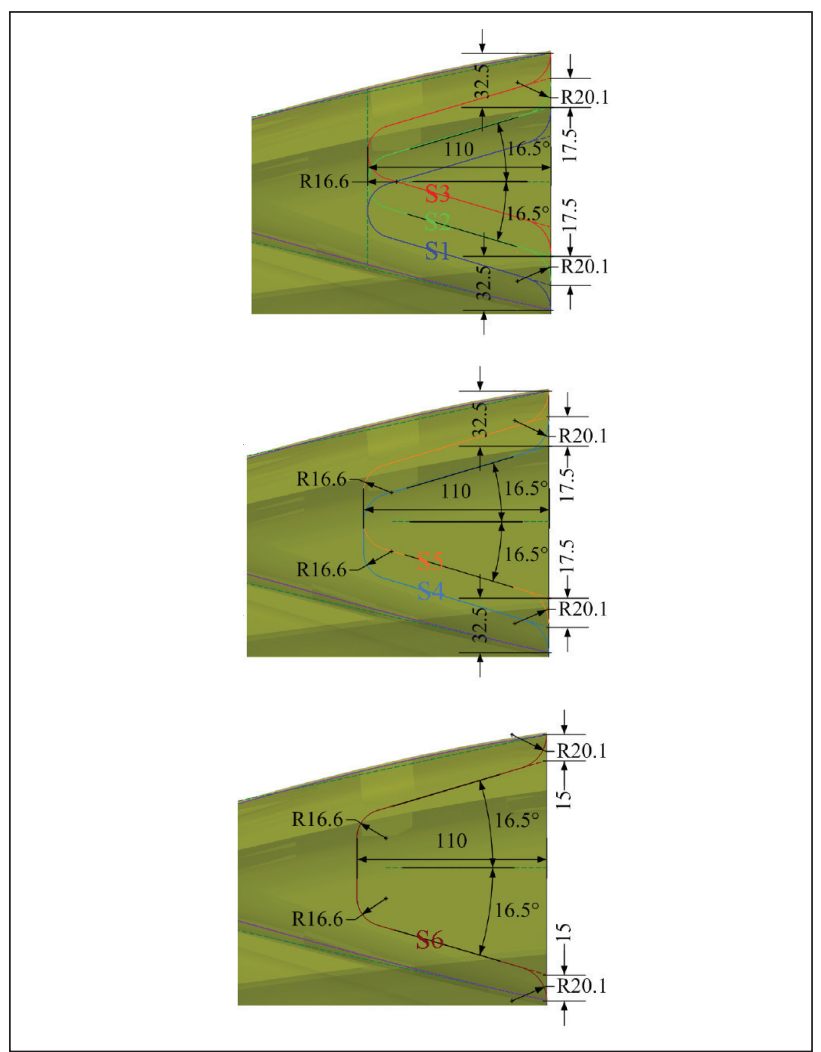

Figure 3. Geometry dimensions of each scalloping alternating lobed nozzle (all dimensions are in $\mathrm{mm}$ ).

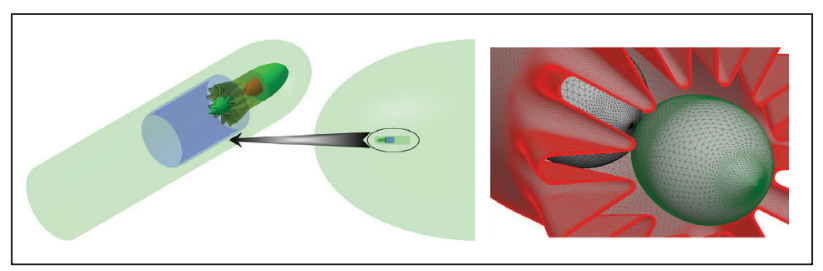

Figure 4. Numerical simulation of flow-field domain and grids.

was $0.05 \mathrm{~mm}$. Also in Fig. 4, a refined domain was adopted near the region where severe changes in the velocity and temperature were expected. In the refined domain, the maximum grid size was about $15 \mathrm{~mm}$, and more than 20 million tetrahedral cells were meshed. Total number of cells in the whole flow-field was more than 23 million.

The flow-field simulation is based on Fluent software packages. Three-dimensional Reynolds averaged N-S equations were solved. According to previous studies (Sheng et al. 2014; Sheng et al. 2015), the most appropriate turbulence model is the SST k- $\omega$. The far-field boundary condition was set to pressure inlet and pressure outlet. The operating pressure was $101,325 \mathrm{~Pa}$, the temperature was $300 \mathrm{~K}$ and the turbulence intensity was set to $5 \%$. The discretized scheme was second-order. The jet inlet velocity was set to $125 \mathrm{~m} / \mathrm{s}$ and temperature was $850 \mathrm{~K}$. Energy equation was involved in the simulation. In this paper, the wall heat transfer and thermal radiation were not coupled, thus the boundary condition of adiabatic wall was used.

\section{CFD MODEL VALIDATION}

To quantify the numerical method, a comparison against the available experimental results ( $\mathrm{Hu}$ et al. 2000, 2005) is performed for the velocity vector and axial velocity distributions. The lobed nozzle in Hu's experiment has six lobes. The width of lobe is $6 \mathrm{~mm}$, and the height of the lobe is $15 \mathrm{~mm}$. The inward and outward lobe penetration angles are $25^{\circ}$ and $14^{\circ}$, respectively. The diameter of the lobed nozzle is $d=40 \mathrm{~mm}$. The jet velocity at the exit of the tested nozzle is set at about $20 \mathrm{~m} / \mathrm{s}$. The Reynolds number of the jet flow, based on the lobed nozzle diameter and the jet velocity, is about 55,000. Particle Image Velocimetry (PIV) system, called Dual-Plane Stereoscopic PIV (DP-SPIV), was used to measure the mixing flow results.

The results are illustrated in Fig. 5, where 20,60 and $80 \mathrm{~mm}$ are the distances from the nozzle exit. It can be seen that the simulated results compare well with the experimental data. Figure 6 displays the mixing decay of the maximum value of the streamwise vorticity in the simulation and Hu's experiment. Except for some differences after the breakdown, the simulated maximum value of the streamwise vorticity has a good agreement. According to the corresponding references (Hu et al. 2000, 2005), the major part of the enhanced mixing caused by the special geometry of the lobed nozzle is concentrated within the first two diameters

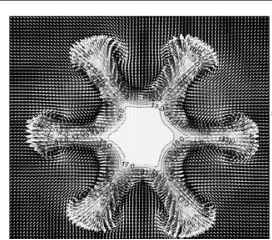

(a) DP-SPIV $20 \mathrm{~mm}$

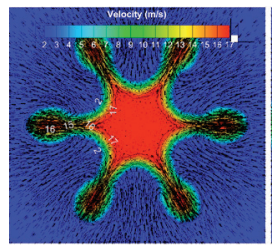

(b) SST k-w $20 \mathrm{~mm}$

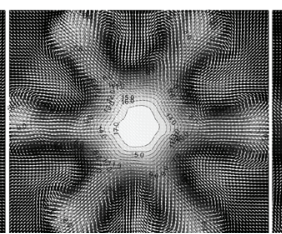

$60 \mathrm{~mm}$

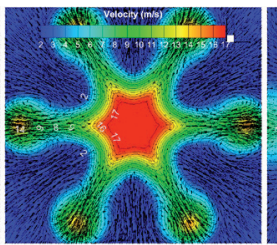

$60 \mathrm{~mm}$

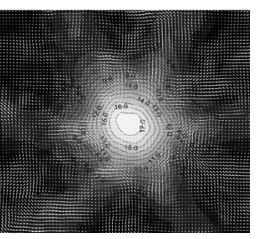

$120 \mathrm{~mm}$

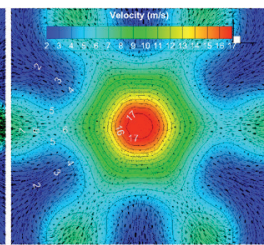

$120 \mathrm{~mm}$
Figure 5. Velocity vector and axial velocity distributions of the six lobes nozzles. Comparison of (a) Experimental data with (b) Numerical results. 
of the lobed nozzle, thus the difference in the maximum value of the streamwise vorticity after the vortices break down only has slight influence on the accuracy of the simulated jet mixing. Therefore, these results validate that the numerical method is suitable for the simulation of the lobed nozzles in this study.

The dimensionless streamwise vorticity $\omega_{x}(\mathrm{Hu}$ et al. 2005) is defined as:

where:

$$
\omega_{x}=\frac{D}{u_{\mathrm{P}}}\left(\frac{\partial w}{\partial y}-\frac{\partial v}{\partial z}\right)
$$

$D$ is the diameter of the mixing duct; $v$ and $w$ are the velocities of mixing stream in the $y$ and $z$ directions; $u_{p}$ is the initial velocity of the primary stream.

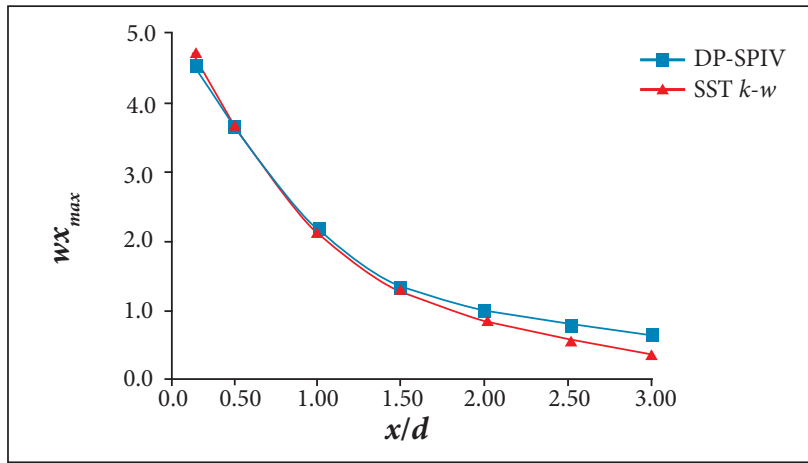

Figure 6. The maximum value of the streamwise vorticity of the lobed nozzle with six lobes.

\section{RESULTS AND DISCUSSION}

\section{JET MIXING FLOW-FIELD ANALYSIS}

Figure 7 shows the axial velocity isolines and temperature cloudscape at $x / d=0.25$ and 0.5 . As can be seen in this figure, the mixing of wave trough is more rapid than the lobe peak and sidewall region for the PLN; with a constant scalloping width, the improved mixing regions of configurations S1, S2 and S3 change on the radial direction with the scalloping position (see Figs. $7 \mathrm{~b}, 7 \mathrm{c}$ and $7 \mathrm{~d}$ ). The improved mixing regions of $S 4$ are almost equal to that of S1; that of S5 is almost equal to that of S2; and that of S6 is almost equal to that of S4; the unmixed primary stream near the lobe peaks of $S 4$ is less than that of S1 but more than that of S2; that of S5 is less than that of S2 but more than that of S3; and that of S6 is less than that of S4 but more than that of S5 (Figs. 7e, 7f, and 7g). As the position of scalloped lower edge kept invariant, the radial position of the accelerated mixing region of sidewalls did not change. The accelerated mixing region is enlarged as scalloped width increased, while the growth rate of enlarged region is less than the growth rate of scalloping width.

\section{STREAMWISE VORTICITY}

Figure 8 shows the non-dimensional streamwise vorticity distribution at $x / d=0.25$ for each lobed nozzle. The streamwise vorticities of S1, S4 and S6 are higher than other scalloped schemes and PLN. One can deduce that inward moving of

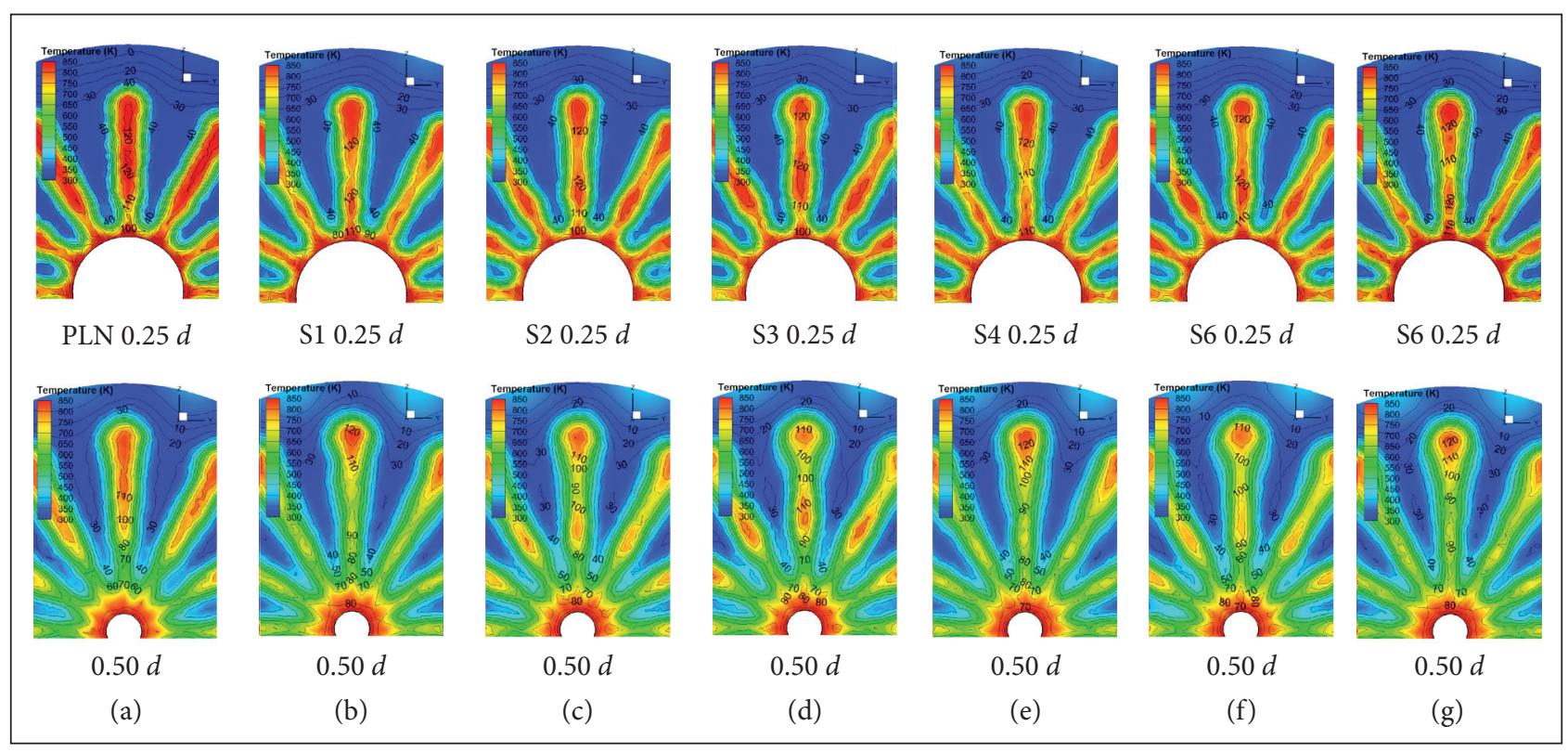

Figure 7. Distributions of axial velocity $(\mathrm{m} / \mathrm{s})$ and temperature at $0.25 d$ and $0.50 d$ for each lobed nozzle. 
scalloped lower edge enhances the effect of strengthening streamwise vortices.

Figure 9 shows the $700 \mathrm{~K}$ temperature iso-surface for each lobed nozzle. The mixing near the sidewalls of PLN is slightly faster than that in the region near the lobe peaks. The scalloping accelerates the mixing in the downstream regions of the lobe peaks and sidewalls, but the complete mixing distance of the primary stream in the core region increases. For the mixing near the sidewalls of all the schemes, the scheme $\mathrm{S} 4$ is faster than S1 and S2; S5 is faster than S2 and S3; S6 has the highest mixing rate in these regions. The mixing rate increases as the scalloping width increases. In the region near the lobe peaks, all scalloped schemes mixing rate are higher than PLN. When the scalloped region with the same width (S1, S2, S3) moves outward in the radial position, the mixing rate increases. The complete mixing distances of the primary stream in the core region for all scalloped schemes are almost the same, except S3, which has slightly shorter complete mixing distance. The scalloping accelerated the mixing of sidewalls region. Part of the mixing air flowed to the core region, and the temperature of secondary flow penetrating into the core region increased. Therefore, the complete mixing distances of the primary stream in the core region increased. The scalloped inner edge of S3 is farther to the core region, less mixing air flows to the core region, and the complete mixing distance of core region for S3 is slightly shorter than other scalloped schemes.

\section{JET MIXING PERFORMANCE}

In the present investigation, the components of primary and secondary streams are treated as the same. The thermal mixing efficiency $\eta_{t r}$ (Xie and Liu 2011) can be expressed as:

$$
\eta_{\mathrm{tr}}=1-\frac{\int\left(T_{\mathrm{m}}-T_{\mathrm{M}}\right)^{2} \mathrm{~d} m_{\mathrm{m}}}{T_{\mathrm{p}}^{2} m_{\mathrm{p}}+T_{\mathrm{s}}^{2} m_{\mathrm{s}}-T_{\mathrm{M}}^{2} m_{\mathrm{m}}}
$$

where:

$m_{p}$ and $m_{s}$ are the mass flux of primary and secondary stream, respectively; $m_{m}$ is the mass flux of the local mixing stream; the parameters $T_{p}$ and $T_{s}$ are the initial temperature of primary and secondary streams; $T_{m}$ is the temperature of the local mixing stream; $T_{M}$ (Xie and Liu 2011) is the temperature for the complete mixing stream of the primary and secondary streams, i.e.:

$$
T_{\mathrm{M}}=\frac{T_{\mathrm{p}} m_{\mathrm{p}}+T_{\mathrm{s}} m_{\mathrm{s}}}{m_{\mathrm{m}}}
$$

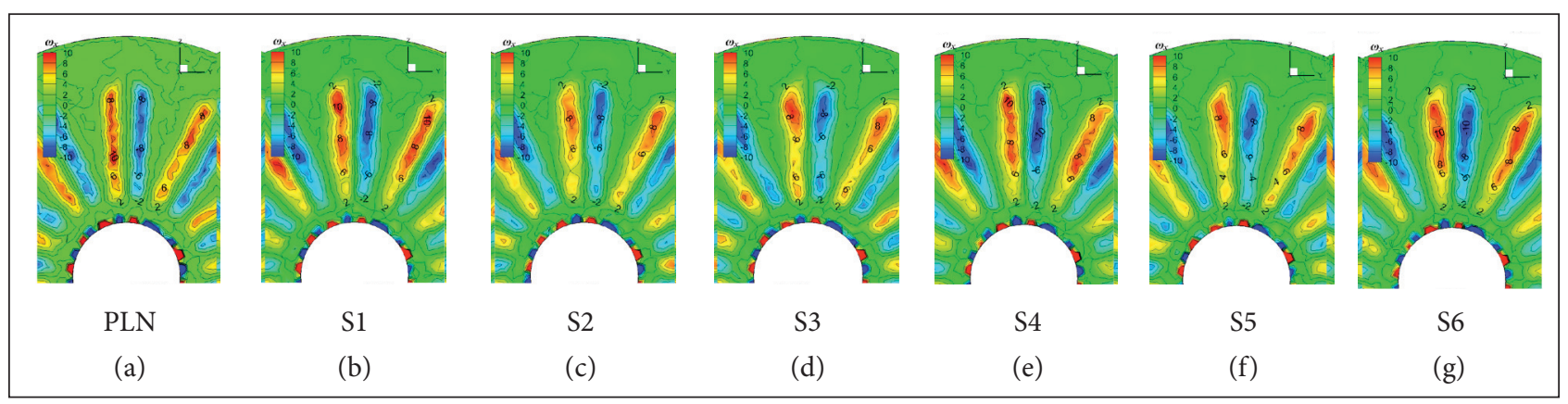

Figure 8. Distributions of the non-dimensional streamwise vorticity at $0.25 d$ for each lobed nozzle.

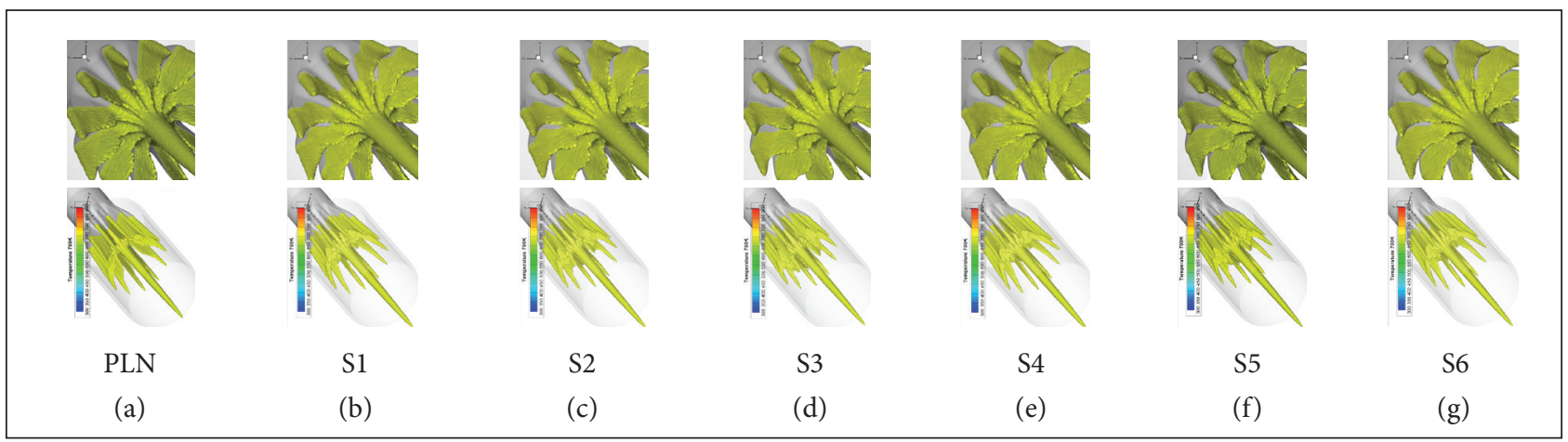

Figure 9. 700K temperature isosurface for each lobed nozzle. 
Thermal mixing efficiency is a vital factor of jet mixing. It indicates the degree of uniform mixing of the primary and secondary streams. The thermal mixing efficiencies against $x / d$ is plotted in Fig. 10, where $x$ is the axial distance measured from the exit of the nozzle. Figure 10 shows that the thermal mixing efficiencies for all the lobed nozzles increase rapidly from $0.25 d$ to $1.0 \mathrm{~d}$ and then slow down. The values for the scalloped schemes are higher than the PLN scheme. In the range of $2.0 d$ to $2.5 d$, only the $\mathrm{S} 3$ scheme index value is higher than the PLN. For the thermal mixing efficiencies of all the scalloped schemes, the values are similar as the scalloped widths kept invariant and increase if the scalloped width is enlarged in the range from $0.25 d$ to $0.5 d$; while in the range of $1.5 d$ to $2.5 d$, the scalloped inner edge is a decisive factor, a higher thermal mixing efficiency can be achieved by the outward moving of scalloped inner edge. All these results indicate that in a short mixing distance, the accelerated mixing of sidewalls region for the scalloped schemes is the primary cause for thermal mixing efficiency improving. As the mixing distance increases, the influence of mixing in the downstream of the lobe peaks and core region becomes more important. As can be seen in Fig. 8 , the streamwise vorticity of S1, S4 and S6 are higher than other scalloped schemes; however, the thermal mixing efficiencies of these schemes do not improve. It can be concluded that the streamwise vortices more intensified by the scalloping do not bring more enhancement on the mixing effectiveness.

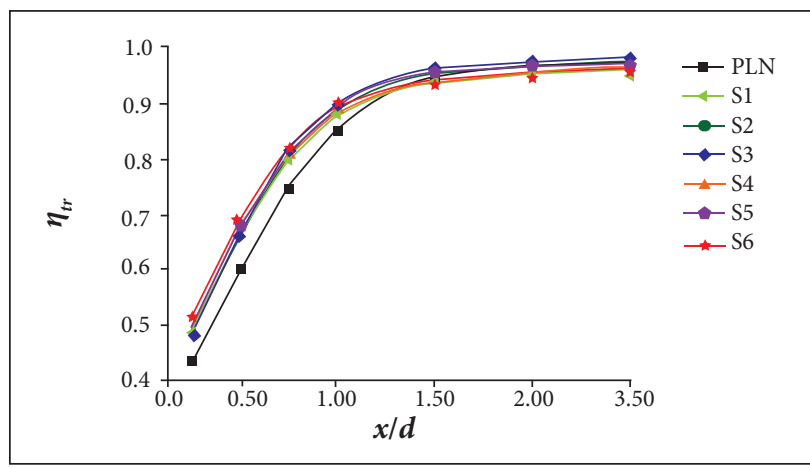

Figure 10. Thermal mixing efficiency along the axis for each lobed nozzle.

\section{THE TOTAL PRESSURE RECOVERY COEFFICIENT}

Another important performance parameter for jet mixing is the total pressure recovery coefficient. A superior total pressure recovery coefficient signifies less energy being consumed in the mixing. The equation is given by (Zhang and Li 2006):

$$
\sigma=\frac{\int P_{\mathrm{m}}^{*} \mathrm{~d} m_{\mathrm{m}}}{\int P_{\mathrm{p}}^{*} \mathrm{~d} m_{\mathrm{p}}+\int P_{\mathrm{s}}^{*} \mathrm{~d} m_{\mathrm{s}}}
$$

where:

$P^{\star}$ is the total pressure of the local mixing stream. The initial total pressure of primary and secondary stream are expressed as $P_{p}^{\star}$ and $P^{\star}$.

It can be seen from Fig. 11 that the total pressure recovery coefficients of cross-sections along the lobed nozzles decreased rapidly up to $1.5 d$, then it slowed down. The coefficients of all scalloped schemes are lower than the PLN scheme between $0.5 d$ and $2.5 d$. The scalloping inner edge is a decisive factor for the total pressure recovery coefficient. Only a slight variation of the total pressure recovery coefficient was found for the configurations S1, S4, S6, in which the inner edge was closely located. The total pressure loss increases as the inner edge moves inside. According to previous analysis (Fig. 8), compared to other schemes, S1, S4 and S6 enhanced the effect of strengthening streamwise vortices more significantly but induced more pressure loss at $0.25 \mathrm{~d}$. Therefore, if the scalloping strengthens streamwise vortices more significantly, more pressure loss will be caused.

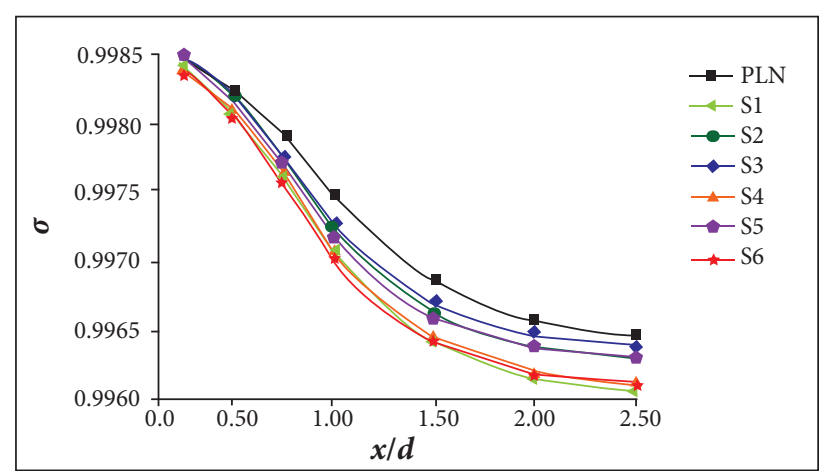

Figure 11. The total pressure recovery coefficients of cross-sections along the lobed nozzles.

\section{CONCLUSIONS}

A series of geometric models of lobed nozzles with central plug were created by different scalloping positions and widths. The shapes of lower and upper edges, cutting depth were kept unchanged. By the use of numerical simulation, the effects of scalloped width and position on the performance of jet mixing in the pumping condition were analyzed. The results are summarized as follows: 
- As the degree of scalloping increased, the mixing in the downstream regions of the lobe peaks and sidewalls were accelerated; however, the primary flow in center needed a larger mixing length. When the position of scalloped lower edge kept invariant, the radial position of the accelerated mixing region of sidewalls did not change.

- When the scalloped region with the same width moved outward in the radial, the mixing rate of downstream lobe peak region increased. The accelerated mixing region was enlarged as scalloped width increased; however, the growth rate of enlarged region was less than the growth rate of scalloping width.

- The inward moving of scalloped lower edge enhanced the effect of strengthening streamwise vortices; however, the strengthened streamwise vortices by the scalloping did not bring higher mixing effectiveness but induced more pressure loss.

\section{REFERENCES}

Abolfadl MA, Sehra AK (1993) Experimental investigation of exhaust system mixers for a high bypass turbofan engine. AIAA-93-0022. Proceedings of the 31st Aerospace Sciences Meeting; Reno, USA.

Acheson KE, Marques EC, Moore MD (2011) A partial mixer nozzle for turbofan nacelles to provide significant jet mixing control. AIAA11-3189. Proceedings of the 29th AIAA Applied Aerodynamics Conference; Honolulu, Hawaii.

Elliott JK, Manning TA, Qiu YJ, Greitzer, EM, Tan CS, Tillman TG (1992) Computational and experimental studies of flow in multi-lobed forced mixers. AlAA-92-3568. Proceedings of the 28th Joint Propulsion Conference and Exhibit; Nashville, USA.

Hu H, Kobayashi T, Saga T, Sage T, Taniguchi, N, Liu HX, Wu SS (1999) Research on the Rectangular Lobed Exhaust Ejector/Mixer Systems. Trans Jpn Soc Aeronaut Space Sci 41(34):187-192.

$\mathrm{Hu}$ H, Kobayashi T, Saga T, Segawa S, Taniguchi N (2000) Particle image velocimetry and planar laser-induced fluorescence measurements on lobed jet mixing flows. Exp Fluids 29:141-157. doi: $10.1007 / \mathrm{s} 003480070016$

$\mathrm{Hu}$ H, Saga T, Kobayashi T (2005) Dual-plane stereoscopic PIV measurements in a lobed jet mixing flow. AIAA-05-0443. Proceedings of the 43rd AIAA Aerospace Sciences Meeting and Exhibit; Reno, USA.

Lei ZJ (2011) Experimental study on the mixing mechanism of lobed mixer with inlet swirl in model turbofan engines ( $\mathrm{PhD}$ thesis). Beijing: University of Chinese Academy of Sciences. In Chinese.

Lei ZJ, Mahallati A, Cunningham M, Germain P (2012) Effects of core flow swirl on the flow characteristics of a scalloped forced mixer. J Eng Gas Turbines Power 134(11):111-201. doi: 10.1115/1.4005968

Wright A, Lei ZJ, Mahallati Ali (2013) Effects of scalloping on the mixing mechanisms of forced mixers with Highly swirling core flow. J Eng Gas Turbines Power 135(7):1-10. doi: 10.1115/1.4024043

Mao R, Yu SCM, Zhou T, Chua LP (2009) On the vorticity characteristics of lobed-forced mixer at different configurations. Exp Fluids 46:10491066. doi: 10.1007/s00348-009-0613-x

McCormick DC, Bennett JC (1994) Vortical and turbulent structure of a lobed mixer free shear layer. AIAA J 32(9):1852-1859. doi: $10.2514 / 3.12183$
Mitchell MG, Smith Ol, Karagozian AR (2004) Passive fuel-air mixing and emissions control via lobed injectors. AIAA J 42(1):61-69.

Pan CX, Zhang JZ, Shan Y (2013) Effects of exhaust temperature on helicopter infrared signature. Appl Therm Eng 51(1-2):529-538. doi: 10.1016/j.applthermaleng.2012.09.016

Povinelli LA, Anderson BH (1984) Investigation of mixing in a turbofan exhaust duct, part II: computer code application and verification. AIAA J 22(4):518-525. doi: 10.2514/3.8433

Presz Jr WM, Reynolds Jr G, McCormick D (1994) Thrust augmentation using mixer-ejector- diffuser systems. AIAA-94-0020. Proceedings of the 32nd Aerospace Sciences Meeting and Exhibit; Reno, USA.

Sheng ZQ, Chen SC, Wu Z, Huang PL (2015) High mixing effectiveness lobed nozzles and mixing mechanisms. Sci China Tech Sci 58:12181233. doi: 10.1007/s11431-015-5846-8

Sheng ZQ, Huang PL, Ji JZ, Wang Y (2014) Effects of modification on jet mixing of Sword Alternating Lobed Nozzle. J Beijing Univer Aeronau Astronau 40(10):1417-1423. doi: 10.13700/j.bh.10015965.2013.0633

Smith LL, Majamaki AJ, Lam IT, Delabroy O, Karagozian AR, Marble FE, Smith Ol (1997) Mixing enhancement in a lobed injector. Phys Fluids 9(3):667-678. doi: 10.1063/1.869224

Xie Y, Liu YH (2011) A modified thermal mixing efficiency and its application to lobed mixer nozzle for aero-engines. Heat Transf Res 42(4):317-335. doi: 10.1615/HeatTransRes.2011003398

Yu SC, Hou Y, Chan WK (2000) Scarfing and scalloping effects on lobed forced mixer at low-speed conditions. J Propul Power 16(3):440448. doi: $10.2514 / 2.5608$

Yu SCM, Yip TH, Liu CY (1997) Mixing characteristics of forced mixers with scalloped lobes. J Propul Power 13(2):305-311. doi: $10.2514 / 2.5164$

Zhang JZ, Li LG (2006) Aeronautic ejector/mixer. Beijing: National Defense Industry Press.

Zhang JZ, Pan CX, Shan Y (2014) Progress in helicopter infrared signature suppression. Chin J Aeronaut 27(2):189-199. doi: 10.1016/j.cja.2014.02.007 